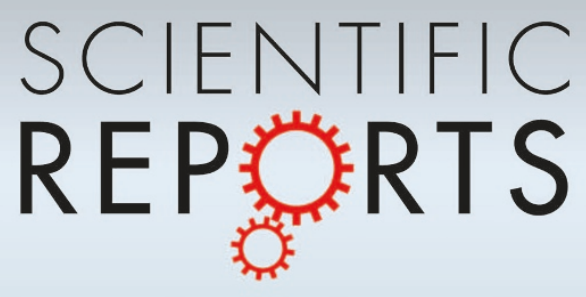

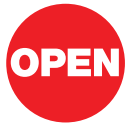

SUBJECT AREAS: OPTICS AND PHOTONICS

TRANSFORMATION OPTICS

FIBRE OPTICS AND OPTICAL COMMUNICATIONS

NONLINEAR OPTICS

Received

18 July 2012

Accepted

19 November 2012

Published

6 December 2012

Correspondence and requests for materials should be addressed to

J.F. (jfatome@ubourgogne.fr)

\section{A universal optical all-fiber omnipolarizer}

\author{
J. Fatome' ', S. Pitois' ', P. Morin' ', E. Assémat' , D. Sugny' ', A. Picozzi ', H. R. Jauslin' ', G. Millot' , V. V. Kozlov²,3 \\ \& S. Wabnitz ${ }^{2}$
}

'Laboratoire Interdisciplinaire Carnot de Bourgogne (ICB), UMR 6303 CNRS - Université de Bourgogne, 9 Av. Alain Savary, BP 47870, 21078 Dijon, France, ${ }^{2}$ Department of Information Engineering, Università di Brescia, Via Branze 38, 25123 Brescia, Italy, ${ }^{3}$ Department of Physics, St.-Petersburg State University, Petrodvoretz, St.-Petersburg, 198504, Russia.

Wherever the polarization properties of a light beam are of concern, polarizers and polarizing beamsplitters (PBS) are indispensable devices in linear-, nonlinear- and quantum-optical schemes. By the very nature of their operation principle, transformation of incoming unpolarized or partially polarized beams through these devices introduces large intensity variations in the fully polarized outcoming beam(s). Such intensity fluctuations are often detrimental, particularly when light is post-processed by nonlinear crystals or other polarization-sensitive optic elements. Here we demonstrate the unexpected capability of light to self-organize its own state-of-polarization, upon propagation in optical fibers, into universal and environmentally robust states, namely right and left circular polarizations. We experimentally validate a novel polarizing device - the Omnipolarizer, which is understood as a nonlinear dual-mode polarizing optical element capable of operating in two modes - as a digital PBS and as an ideal polarizer. Switching between the two modes of operation requires changing beam's intensity.



he state of polarization is one of the three characteristics of an electromagnetic wave, the others being its energy (or photon number) and frequency. Although the management and measurement of the wave energy and frequency have progressed throughout the laser era up to unprecedented levels of precision, the state of polarization of light still remains largely elusive to control. Indeed, despite the significant progress in optical fiber manufacturing, because of residual birefringence or strain the polarization of light remains unpredictable after propagating a few hundred meters in a fiber. Moreover, in spite of the recent tremendous technological developments in waveguide and fiber-based polarization controllers ${ }^{1,2}$, the basic principle of operation of these devices rests upon a combative strategy, consisting in linear polarization transformations followed by partial diagnostic associated with an active loop control driven by complex algorithms ${ }^{1,2}$. Here, we propose a radically different approach which is based on preventing polarization fluctuations in optical fibers, rather that post-compensating them. Consider for example the action of a linear polarizer and a polarization beam-splitter (PBS) on a sequence of three perfectly polarized pulses, as shown in Fig. 1a,c. The polarizer only passes one component of polarization (SOP) and rejects the other ones. As a result of this rejection principle, in the example of Fig. 1a only two pulses out of three pass through the polarizer (albeit with largely different peak powers), while the last pulse with orthogonal polarization is blocked and removed from the outcoming beam. Hence, due to the rejection principle, all input polarization fluctuations are transformed into output intensity variations, introducing a large Relative-IntensityNoise (RIN); even if the input beam had a steady intensity in time. Indeed, this property is intrinsic to linear polarizers or any device exhibiting strong polarization-dependent losses. It may seem that a linear PBS is free of RIN because the device is lossless, i.e. the total energy of the transmitted beam is conserved. This is not true clearly RIN is still present in each individual output channel, as illustrated by Fig. 1c. The principle of operation of a linear PBS (which we call here the principle of continuous splitting), rules that the last pulse must be split in nearly equal portions between the two output channels. Therefore, the peak intensities of pulses in each output stream are modulated in time, even though the pulses had equal intensities in the stream incident onto the PBS.

Here we propose, experimentally validate and theoretically describe the so far unexpected capability of light to self-organize its SOP in optical fibers, with no need for additional control elements. We baptize this new device as the Omnipolarizer. The principle of operation of our Omnipolarizer is schematically illustrated in Fig. 2. Basically, the Omnipolarizer is simply composed by a single span of nonlinear telecom fiber, where a signal beam nonlinearly interacts through a four-wave-mixing process with its own counter-propagating replica produced by means of a back-reflection at the fiber output, see Fig. 2a. Depending on the mirror reflection coefficient 
a

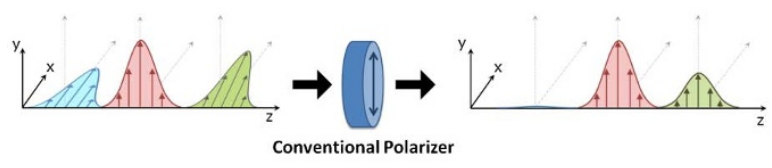

b

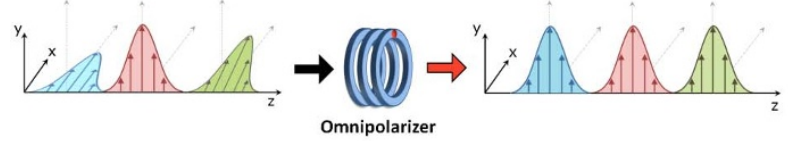

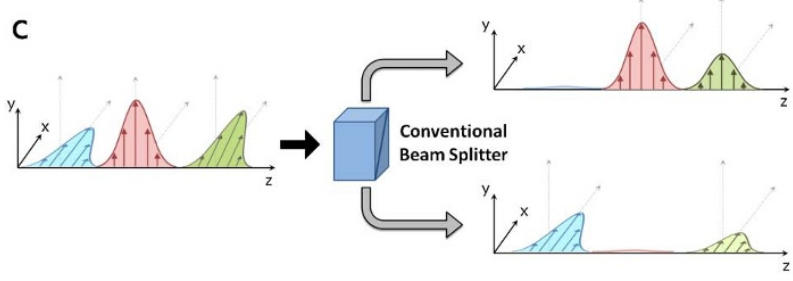

d

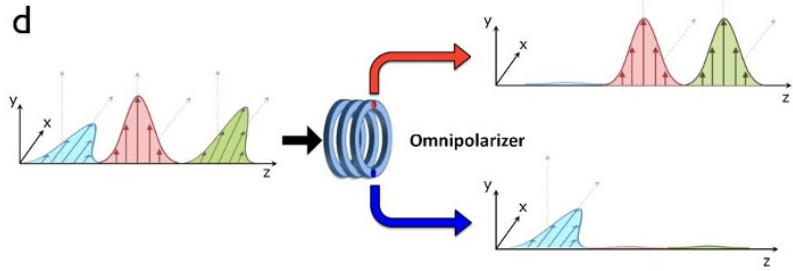

Figure $1 \mid(\mathrm{a}, \mathrm{b})$ Illustration of the lossy nature of a linear polarizer based on the rejection principle, as opposed to the Omnipolarizer. Three input pulses, with different initial polarization states are incident on a) the linear (or conventional) passive polarizer; b) the Omnipolarizer. In the first case, we observe strong output intensity variations due to the rejection principle. In the second case all three pulses pass through with no degradation. In both cases the outcoming pulses are vertically polarized. (c, d) Illustration of the continuous splitting principle of the linear (or conventional) PBS, as opposed to the discrete splitting principle of the Omnipolarizer. Three pulses, with different initial polarization states are incident on c) the linear PBS; d) the Omnipolarizer. In the first case, pulse splitting and intensity variations are observed on each axis of the PBS. In the second case, no splitting is observed: all energy is routed to either one or another channel, simply depending on the initial polarization ellipticity of the pulse. Note that this figure only serves for illustrative purposes. Indeed, the Omnipolarizer splits mutually orthogonal circular (and not linear, as shown in the figure) polarizations.

(which can be even greater than unity when the back-propagating signal is amplified into a reflective-loop, see Fig. 2b), the Omnipolarizer may operate in (and can be switched among) two distinct RIN-free regimes. Consider first the PBS mode, which is obtained with a below-unity back-reflection coefficient. For any arbitrary polarized input signal, two fixed output SOPs are produced, corresponding to right or left circular states (i.e., the two poles of the
Poincaré sphere). These SOPs are universal as they do not depend on the telecom fiber sample or environmental conditions. The sign of the initial signal ellipticity determines which of the two poles is obtained at the device output. Next consider the polarizer mode which is obtained when the back-reflection coefficient is larger than unity (i.e., the back-reflected light is amplified). In this case a single circular output SOP is generated, irrespective of the initial

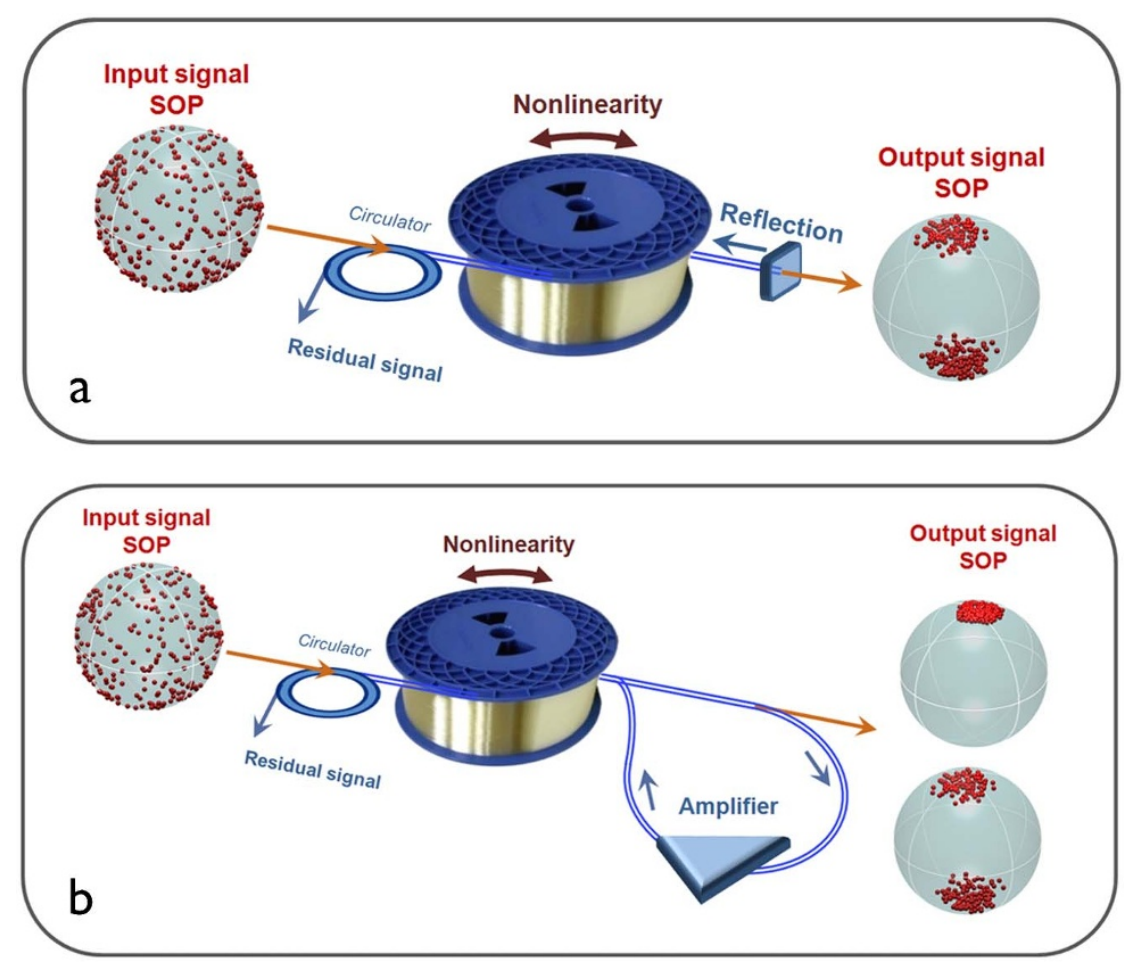

Figure $2 \mid$ Schematics and principle of the Omnipolarizer. (a) Passive setup: the light beam interacts nonlinearly in an optical fiber with its backward replica, obtained by inserting a partially reflecting mirror. The device behaves as a discrete PBS. Depending on its initial ellipticity, the input signal is digitally routed towards one or the other pole of the Poincaré sphere, corresponding to circular polarization states. (b) Active setup: the back-reflected signal is amplified in a reflective fiber loop (circulator \& amplifier). The Omnipolarizer can switch between the PBS and polarizer modes depending on the back-reflected power. In the polarizer mode, all the input SOPs remain trapped around a small spot on the Poincare sphere. 
polarization state of the incoming signal. As we shall see, the major advantage of the Omnipolarizer is not just that two polarization control functions may be combined into a single optical device, but rather in the peculiar mode of operation of both the PBS and the nonlinear polarizer.

Let us first characterize the nonlinear polarizer function of the Omnipolarizer and contrast its operation principle with the rejection principle of a linear polarizer, cf. Fig. 1a and Fig. 1b. In the Omnipolarizer, all incident SOPs are nonlinearly trapped and merge into a small spot around a well-defined SOP at its output. Therefore, this device is free of RIN - a steady in time and varying in polarization input beam is converted into a steady in time and fully polarized output beam. Note however that the Omnipolarizer cannot remove any RIN existing originally in the intensity profile of a signal. Yet, unlike conventional passive linear polarizers, the device does not introduce any additional RIN penalty due to the polarization rejection principle and propagation channel. Next, consider the PBS feature of the Omnipolarizer and contrast its discrete splitting operation principle with the continuous splitting which is inherent to a linear PBS. Depending on its input polarization ellipticity, the signal beam is directed into either one or another channel at the device output. This means that an input pulse is no longer split among the two output channels in amounts which are proportional to the projections of its SOP on the two polarization axes of the PBS. To the contrary, the signal pulse is routed to an output channel as a single digital entity, cf. Fig. $1 \mathrm{~b}$ and Fig. 1d. Therefore we may conclude that this device, when functioning as a PBS (called here discrete PBS) is free from RIN.

In the quest of more transparency for future optical networks, there is a need for ultrafast all-optical control of light polarization, which has motivated a renewed interest in polarization optics. In this field research moves along three distinct paths: i) the development of linear and nonlinear methods of re-polarization of a partially coherent and initially fully depolarized light with interferometers ${ }^{1}$, in a random scattering medium ${ }^{4}$, via propagation in a Kerr medium ${ }^{5-9}$, or even through propagation of a diffracting beam in free space ${ }^{10}$; ii) the engineering of dissipative polarizers ${ }^{11-18}$; iii) the design of nonlinear methods of lossless polarization control of fully coherent and depolarized beams ${ }^{19-23}$. Polarizers belonging to the first class are based on the coherent suppression of phase noise, which is intimately related to the incoherent nature of light beams. These polarizers are not of interest to us here, because they either are inapplicable to fully coherent beams, or when applicable they are not free from RIN. Examples of devices belonging to the second class are the Brillouin amplifiers ${ }^{11-14}$ proposed by L. Thevenaz et al., and Raman amplifiers ${ }^{15-18}$ first demonstrated by M. Martinelli et al. in ref. 15. These devices do not conserve the energy of the beam and, more importantly, because of their intrinsic properties based on polarization dependent gain, they suffer from a large amount of output RIN. Our Omnipolarizer belongs to the third class. Polarizers belonging to this class are all free of RIN. However most of these devices have the drawback of requiring an additional polarization control intense beam (pump beam), whose state of polarization must be in turn also accurately stabilized $^{19-22}$. In contrast, the Omnipolarizer does not need any pump beam or active opto-electronic element, since light is capable of selforganizing its own SOP. Its operation principle is based on the ultrafast Kerr nonlinearity inherent to silica glass, and it is at least six orders of magnitude faster ${ }^{24}$ than the single-beam polarizers utilizing the slow photorefractive nonlinearity which were pioneered $\mathrm{in}^{23}$.

Here, the Omnipolarizer is not only a fast single-beam polarizer free of RIN, but most importantly it also demonstrates a RIN-free PBS based on the discrete splitting principle, as exemplified by Fig. 1d. From a fundamental point of view, the experimental demonstration of the Omnipolarizer represents a significant step forward as its operation goes beyond current way of thinking. Indeed, this device provides the first clear experimental demonstration example of the self-organization of light polarization in a nonlinear medium thanks to feedback reflection, which is described by means of a perfectly isotropic model (see Eq. (1) and Ref. 25). Similarly to the spontaneous organization which may occur in the spatial domain as it was numerically predicted in ref. 26, in spite of its symmetry the medium prefers to support a strongly anisotropic polarization pattern - one or two tightly localized spots on the Poincare sphere for the output signal SOP (see Fig. 2) instead of the expected isotropic distribution, i.e. a uniform coverage of the Poincaré sphere, see Fig. 2. The illustration of the beam SOPs on the Poincaré sphere in Fig. 2 comes handy to visualize the main function of the Omnipolarizer - the spontaneous re-polarization of an initially unpolarized wave.

\section{Results}

We implemented and demonstrated the concept of universal Omnipolarizer as shown in Fig. 3, see the Methods section for additional details. The efficiency of the device was first characterized when operating in the discrete PBS mode. In this case the setup only involves an output reflective element (i.e., the configuration depicted in Fig. 2a), such as a Fiber Bragg Grating (FBG, used here with 95\% of reflection, $5 \%$ of transmission) or an optical circulator plus a fiber coupler, or end fiber coating. The corresponding experimental results are provided in Fig. 4. The SOP of an initial On/Off Keying (OOK) 40-Gbit/s signal centred around $1564 \mathrm{~nm}$ was scrambled so that it spreads over the entire Poincaré sphere. As a result, its eyediagram signal was completely closed beyond a linear polarizer.

When the input average power was increased up to $27 \mathrm{dBm}$, we could observe that the output SOP self-stabilized in two orthogonal points on the Poincare sphere: light self-organized its SOP. Indeed, for a positive (negative) ellipticity of the input signal SOP (i.e., the red (blue) points in Fig. 4), the output signal SOP remained confined around the north (south) pole of the sphere, that is to say close to the right (left)-handed circular polarization. In order to quantify the quality of the attraction process, the residual fluctuations in the output SOP can be contained in a maximum circle-cap characterized by a solid angle of $0.42 \mathrm{sr}$ for the north pole ( $0.68 \mathrm{sr}$ for the south), corresponding to a maximum fluctuation of the ellipticity angle of $0.37 \mathrm{rad}(0.47 \mathrm{rad})$ peak-to-peak or a solid angle of $0.10 \mathrm{sr}(0.12 \mathrm{sr})$ in rms corresponding to a variation of the ellipticity angle of $0.18 \mathrm{rad}$ $(0.20 \mathrm{rad})$, respectively. This residual distribution around a small area is attributed to the fact that the process of self-organization asymptotically converges to the poles in the limit case of very long fibers or high input powers. Consequently, nearly orthogonal input SOPs are difficult to attract into a single output point. Moreover, it is important to note that the two output SOPs are universal, in the sense that they do not depend on either the input signal, the telecom fiber sample, the laboratory reference frame or any environmental changes. Indeed, we checked that straining the fiber does not influence the position and width of the output SOP distributions. Moreover, by means of an air-free quarter-wave plate/polarizer setup, we measured the absolute (i.e., in the fixed laboratory frame) value of the SOP which always remains in a circular state. As a result, in the configuration leading to two universal output SOPs the Omnipolarizer behaves as a discrete PBS. Indeed, in spite of the initial polarization scrambling, no intensity fluctuations can be observed in the eye-diagrams of the $40-\mathrm{Gbit} / \mathrm{s}$ output intensity profiles. Depending on its initial ellipticity, all of the 40-Gbit/s signal energy is digitally routed to either the right or left-circular SOP without any pulse splitting.

Next we tested the second configuration shown in Fig. 2b and 3, which involves the reflective loop set-up and allows the Omnipolarizer to operate in (or switch among) the two different regimes - a polarizer or a PBS. Namely, one or two points of SOP stabilization can be observed, depending on the amount of energy that returns back into the fiber upon reflection. An associated short movie, which can be found in the Supplementary Movie, illustrates 


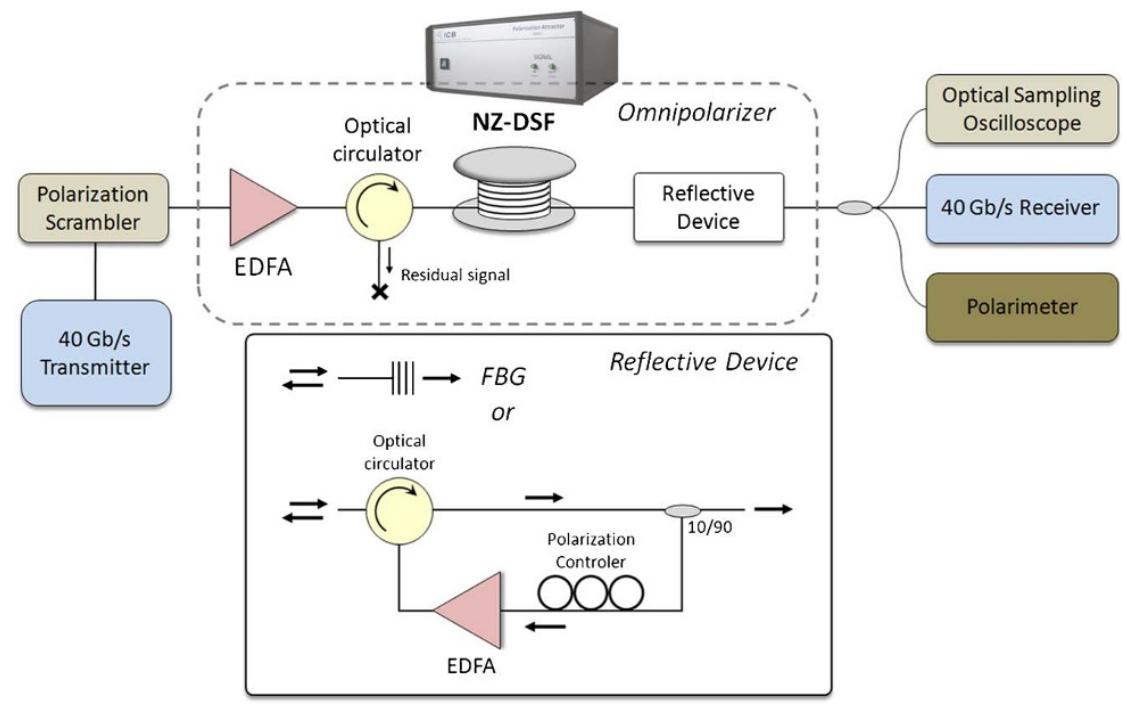

Figure 3 Experimental set-up of the Omnipolarizer. The polarization state of an input 40-Gbit/s Return-to-Zero transmitter (Tx) is first randomly distributed onto the Poincare sphere by means of a polarization scrambler. Next the signal is amplified by means of an Erbium doped fiber amplifier (EDFA) up to $27 \mathrm{dBm}$ before injection into a 6.2-km long standard silica Non-Zero Dispersion-Shifted Fiber (NZ-DSF). After propagation, the signal is back-reflected by either a Fiber Bragg Grating (FBG) or an amplified reflective loop consisting of a circulator, a fiber coupler to collect the output signal, and a second EDFA, respectively. At the receiver, the efficiency of the self-repolarization effect is evaluated onto the Poincaré sphere. Moreover, timedomain monitoring of the output SOP is obtained by means of eye-diagrams and BER measurements of the signal passing through a conventional linear polarizer. (see Methods for additional experimental details.)

well the evolution of the output SOP as a function of the average power of the reflected signal for an input power of $27 \mathrm{dBm}$. The transition between the two regimes may be clearly observed.

The 40-Gbit/s experimental results obtained for the Omnipolarizer in polarizer mode are summarized in Fig. 5. When the back-reflected signal was amplified by means of the reflective loop configuration of Fig. 3 so that its power was just beyond the input power (i.e., when the power of the back-reflected signal was increased up to $28 \mathrm{dBm}$ ), a single point of stabilization survived: all of the output SOPs remained localized around a small area of the Poincaré sphere (see Fig. 5) contained in a circle-cap characterized by a solid angle of $0.28 \mathrm{sr}$, corresponding to a maximum fluctuation

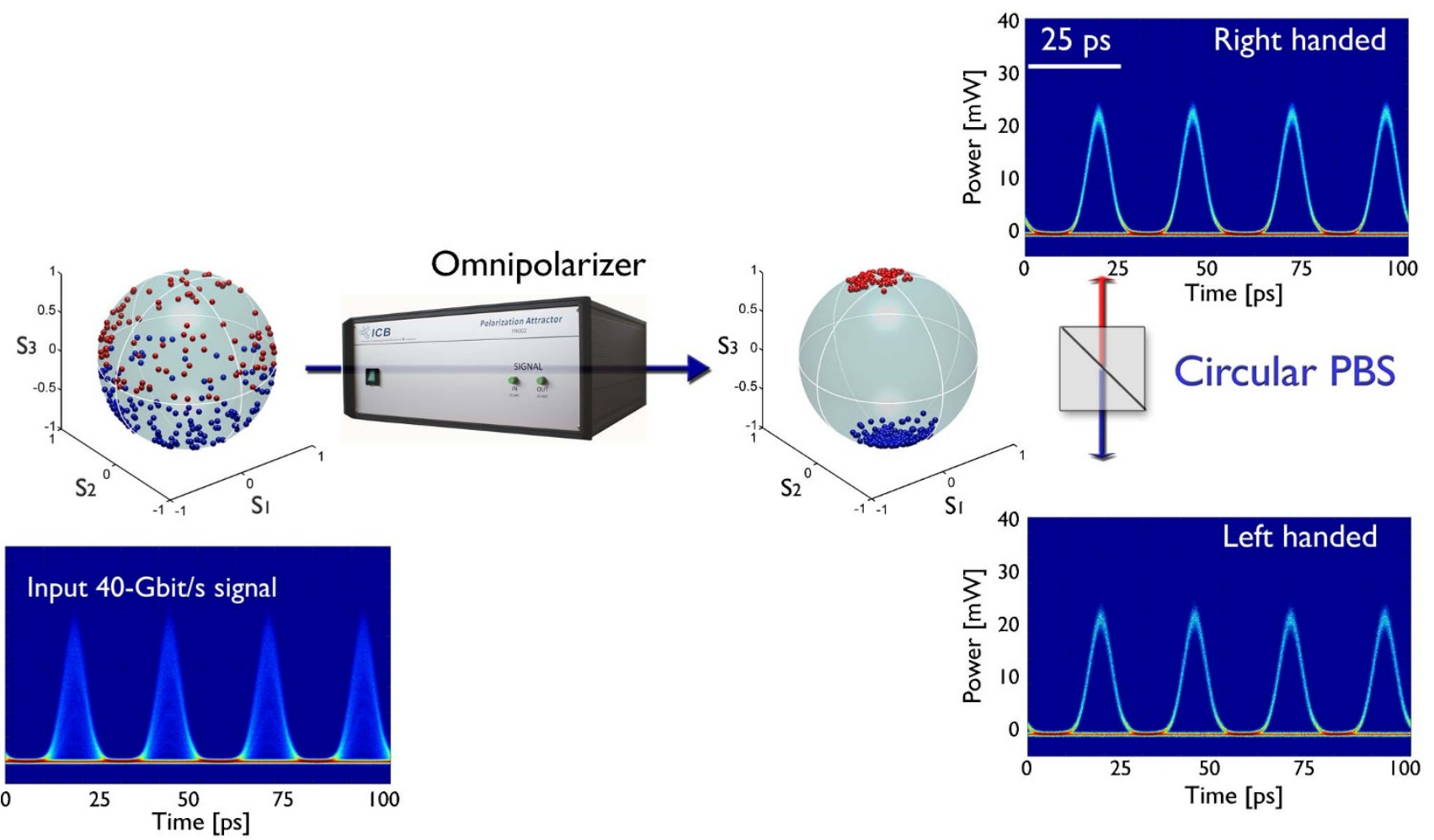

Figure 4 Experimental results obtained in the digital PBS operation mode, in configuration with a FBG, when the input power was set to $27 \mathrm{dBm}$. Two orthogonal universal points of attraction are observed depending on the input signal SOP ellipticity. All initial SOPs initially situated in the northern (southern) hemisphere emerge from the fiber in the right (left) circular polarization, as in a discrete circular PBS. 

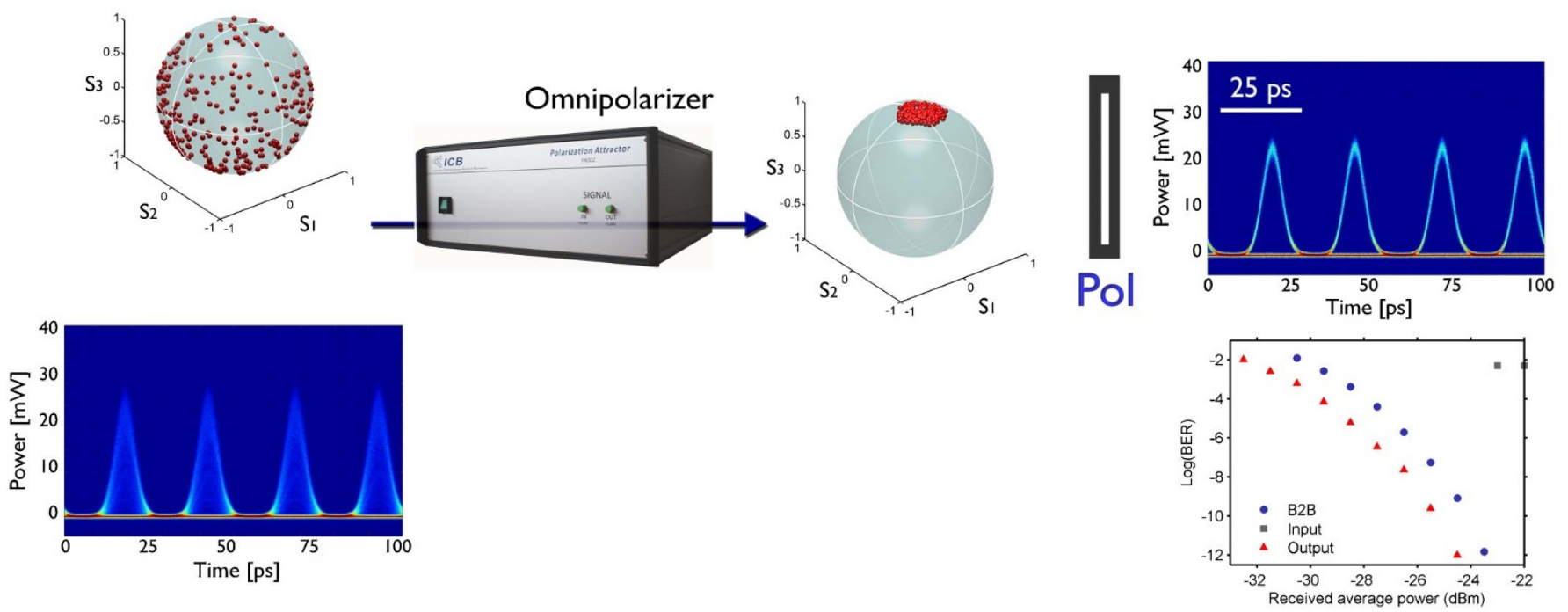

Figure 5 Experimental results obtained in the polarizer mode when the back-reflected signal power is amplified ( $28 \mathrm{dBm})$ just beyond the input one $(27 \mathrm{dBm})$, in a configuration with a reflective loop (Fig.2b). A unique point of stabilization was observed on the Poincare sphere, i.e. the device operates as a polarizer. Fig. 5 illustrates the experimental eye-diagram of the $40-\mathrm{Gbit} / \mathrm{s}$ signal at the input and output of the device through a polarizer. The SOP of the signal is aligned with the polarizer in order to transfer all polarization fluctuations into the intensity domain. We also show BER measurements obtained at the input and output of the Omnipolarizer in the presence of polarization scrambling.

of the ellipticity angle of 0.30 rad peak-to-peak or a solid angle of $0.06 \mathrm{sr}$ in $\mathrm{rms}$ corresponding to a variation of the ellipticity angle of $0.14 \mathrm{rad}$. As in the previous PBS case, we would like to notice that since all the input SOPs converge asymptotically to the pole of the sphere, a large number of nonlinear lengths would be theoretically required to reduce this small area to a single point, which is hardly possible in practice. Note that the finite size of the SOP spot can also be interpreted theoretically by the fact that the singularities lie on the boundary of the energy-momentum diagram (see Fig. 2 of the supplementary materials), so that convergence to the singularities cannot take place in an isotropic way, which limits the efficiency of the attraction process ${ }^{27}$. We remark that the polarization controller inserted into the reflective loop of Fig. 3 can be used to tune the point of attraction on the Poincaré sphere and thus to select a specific output SOP on demand. Consequently, whatever the initial SOP, the output polarization of the 40-Gbit/s signal remains trapped close to a single SOP and the output eye-diagram is completely open behind a polarizer. In other words, a negligible polarization-dependent loss is obtained from the Omnipolarizer, indicating that the selfpolarization process operates in its full strength, free from RIN. More importantly, the corresponding bit-error-rate (BER) measurements (also presented in Fig. 5) show that, in spite of the initial polarization scrambling process, the Omnipolarizer enables clean error-free data recovery behind a polarization dependent component. This completes the demonstration of the powerful SOP stabilization which is achieved by the device.

Theoretical analysis. From a theoretical point of view, the description of the previously discussed self-polarization phenomenon can be established on the analysis of the spatio-temporal evolution of counter-propagating beams (the propagating signal and its own reflective replica) in a standard randomly birefringent telecom fiber, whose dynamics can be modelled by a set of 2 coupled equation $s^{25}$ :

$$
\left\{\begin{array}{l}
\frac{\partial \vec{S}}{\partial t}-v \frac{\partial \vec{S}}{\partial z}=\gamma \vec{S} \times(I \vec{J}) \\
\frac{\partial \vec{J}}{\partial t}+v \frac{\partial \vec{J}}{\partial z}=\gamma \vec{J} \times(I \vec{S})
\end{array}\right.
$$

where $z$ is the spatial coordinate along the fiber, $v$ is the groupvelocity, $\gamma$ is the nonlinear Kerr coefficient, $\times$ denotes the vector product and $I$ is a diagonal matrix with coefficients $(-1,-1,1)^{25}$. The SOPs of the forward and backward beams are described by the Stokes vectors $\vec{S}=\left(S_{x}, S_{y}, S_{z}\right)$ and $\vec{J}=\left(J_{x}, J_{y}, J_{z}\right)$. In spite of its apparent simplicity, model (1) captures all of the essential properties of the Omnipolarizer: excellent quantitative agreement with the experimental results is obtained without using adjustable parameters (see the theoretical supplementary material). In particular, the simulations of Eq. (1) confirm the existence of either one or two points of self-organization for the SOP on the poles of the Poincaré sphere (see Figs. $6 \mathrm{a}-\mathrm{c}$ ). The self-organisation mechanism can be qualitatively understood as follows. Because of statistical averaging over its random linear birefringence distribution, the fiber does not favour any particular polarization direction. For instance, this argument indicates that the SOPs of the two beams cannot relax towards, e.g., a linear state, because such a relaxation would violate the symmetry properties of the fiber. In this respect, the left and right circular SOPs are the only ones which satisfy the requirements imposed by the characteristics of the optical fiber. In its passive configuration, the two circular SOPs are completely symmetric, hence the fiber exhibits two distinct points of attraction for the beam SOPs. The switching between the two modes of operation results from the symmetry-breaking induced by the boundary conditions inherent to the active configuration. More precisely, suppose that the energy of the transmitted signal remains larger than its backward replica (i.e., when a FBG only is used in the configuration of Fig. 2a). In this case the numerical simulations confirm well the experimental observation that, depending on the initial ellipticity of the input SOP, the output SOP self-stabilizes in either one or another of the two poles of the Poincaré sphere (see Fig. 6a). In this situation, the Omnipolarizer acts as a digital PBS, just as in the experimental observations of Fig. 4. On the other hand, whenever the energy of the back-reflected signal becomes equal or slightly larger than the transmitted signal energy, by means of an additional gain as in Fig. 2b, the solutions of Eqs. (1) confirm the experimental findings of Fig. 5 that the Omnipolarizer acts as lossless polarizer (see Fig. 6b). In this case, irrespective of the initial SOP at the input of the device, one obtains at the Omnipolarizer output a unique polarization state without any residual polarization 

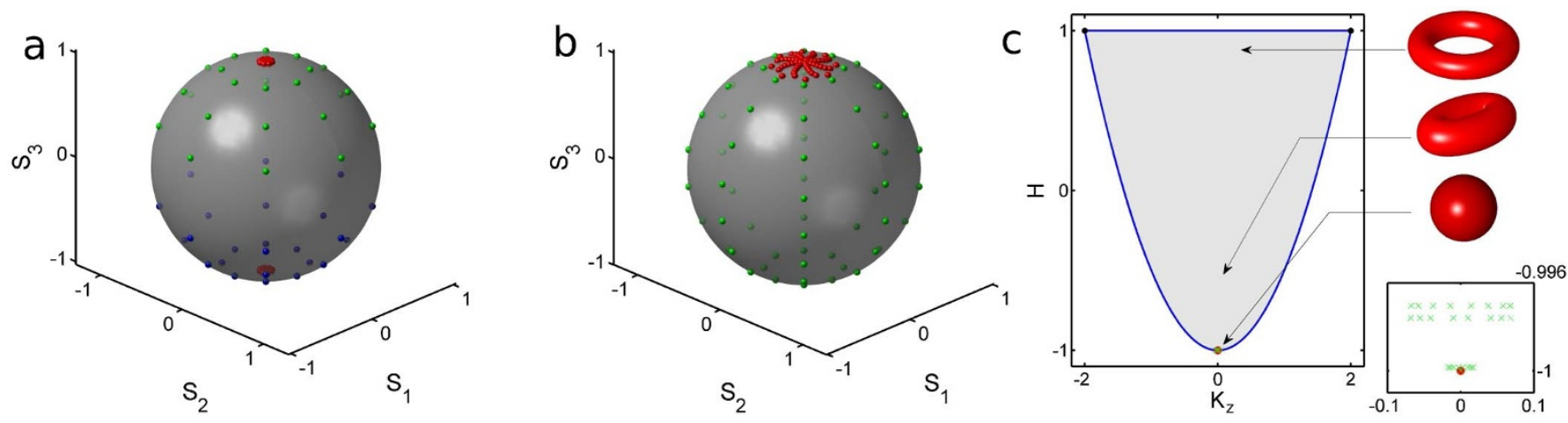

Figure $6 \mid$ (a) Theoretical Poincaré representation obtained by numerically solving the spatio-temporal SOP evolution defined by Eq. (1) in the same configuration as in Fig. 2a involving two output SOP attraction points. To simulate the experiments, we considered a set of 64 different input signal SOPs, uniformly distributed over the Poincaré sphere (blue and green points). The red dots represent output SOPs. The input SOP ellipticity determines the two basins of attraction of the Omnipolarizer: green (blue) dots are attracted to the north (south) pole of the Poincaré sphere. (b)Same as in (a), but in the configuration of Fig. 2b involving a single output SOP attraction point (see the theoretical supplement material for details on the parameters used in the numerical computations). (c) Reduced phase-space representation of the stationary states of the system (1), where the Hamiltonian $\mathrm{H}=\gamma\left(\mathrm{S}_{\mathrm{x}} \mathrm{J}_{\mathrm{x}}+\mathrm{S}_{\mathrm{y}} \mathrm{J}_{\mathrm{y}}-\right.$ $\left.\mathrm{S}_{\mathrm{z}} \mathrm{J}_{\mathrm{z}}\right)$ and $\mathrm{K}_{\mathrm{z}}=\mathrm{S}_{\mathrm{z}}-\mathrm{J}_{\mathrm{z}}$ are conserved quantities. Each point of the phase-space diagram $\left(\mathrm{H}, \mathrm{K}_{\mathrm{z}}\right)$ refers to a torus. The final points of the numerical simulations of Eqs. (1) are represented by green crosses. The small insert is a zoom near the singular point $\left(H=-\gamma, \mathrm{K}_{\mathrm{z}}=0\right.$ : red point), which reveals that the SOP is attracted towards this point. The parameter $\gamma$ has been fixed to 1 in the numerical computations.

depending loss. It can be shown that the selection of either the right or left circular SOP is determined by the angle of polarization rotation in the reflection process. Such angle is at the origin of the symmetry breaking between the two circular SOP, and it can be adjusted experimentally thanks to the polarization controller. More precisely, if one only considers a polarization rotation around the vertical axis of the Poincaré sphere, then a positive (negative) rotation angle favors the left (right) circular SOP. This result can be interpreted intuitively, since a positive angle turns the polarization state in the same sense as the left circular polarization state, which thus favors the attraction process toward this particular SOP (see the theoretical supplementary material).

In addition to this qualitative understanding, the physical mechanism underlying the observed self-polarization phenomenon can be described in terms of mathematical techniques associated with Hamiltonian geometric singularities ${ }^{28}$ (see the theoretical supplemental). Such singularities are topological structures that are also known as singular tori: these can be viewed as a two-dimensional extension of the concept of separatrix, which is a well-known property of basic one-dimensional physical systems (e.g., a pendulum). It can be shown that singular tori play the role of attractors for the SOP as described by Eqs. $(1)^{29,30}$. This is schematically illustrated in Fig. 6c, which provides a phase-space representation of the system: The numerical simulations show that the SOP converges toward the singular torus, i.e. the red point in Fig. 6c. The singular torus for the present model can be represented as a sphere, an object which is topologically singular in the sense that it cannot be transformed into a regular torus by means of continuous deformations.

We briefly illustrate here the results for the passive configuration of the Omnipolarizer and refer the reader to the Supplemental for details. The numerical simulations of Eqs. (1) reveal that, irrespective of the initial conditions, the spatiotemporal dynamics exhibit a relaxation toward a stationary state. This can be understood intuitively by remarking that light reflection on the mirror introduces a supplementary condition for the polarizations states of the forward and reflected waves: $S(L, t)-J(L, t)=0$ at any time $t$. This condition may be viewed as a temporal fixed point on the mirror at $\mathrm{z}=\mathrm{L}$. Then the physical picture that one may have in mind is that the system gradually extends this stationary behaviour from the mirror toward the whole fiber. This relaxation process is possible thanks to the reflected wave, which can evacuate the fluctuations of the waves throughout the free boundary condition at $z=0$. In this way the counter-propagating waves relax toward an inhomogeneous stationary state in which their SOPs keep a fixed constant value for any $\mathrm{z}$.

This brings us to the study of the stationary system (1), whose main property is that it is Hamiltonian with $\mathrm{H}=\gamma\left(\mathrm{S}_{\mathrm{x}} \mathrm{J}_{\mathrm{x}}+\mathrm{S}_{\mathrm{y}} \mathrm{J}_{\mathrm{y}}-\right.$ $\mathrm{S}_{\mathrm{z}} \mathrm{J}_{\mathrm{z}}$ ). In this expression of $\mathrm{H}$, the three axes play an analogous role, a property which leads to three additional conserved quantities, $\mathrm{K}_{\mathrm{x}}=$ $\mathrm{S}_{\mathrm{x}}+\mathrm{J}_{\mathrm{x}}, \mathrm{K}_{\mathrm{y}}=\mathrm{S}_{\mathrm{y}}+\mathrm{J}_{\mathrm{y}}$ and $\mathrm{K}_{\mathrm{z}}=\mathrm{S}_{\mathrm{z}}-\mathrm{J}_{\mathrm{z}}$. These functions play a similar role in the system dynamics, so that all stationary states can be described in a reduced two-dimensional phase-space representation, as illustrated in Fig. $6 \mathrm{c}$ for $\mathrm{H}$ vs $\mathrm{K}_{\mathrm{z}}$. We show in the Supplemental that the singular torus of interest is located at the origin of the diagram at $\mathrm{K}_{\mathrm{y}}=\mathrm{K}_{\mathrm{x}}=\mathrm{K}_{\mathrm{z}}=0$. These values of the constant of the motion together with the mirror boundary conditions impose that only the circular SOPs can exist on the singular torus. In conclusion, the topological properties of the singular torus impose the universal circular SOPs which are selected by the Omnipolarizer.

\section{Discussion}

From the practical side, it is important to answer the question - what is the response time of our Omnipolarizer? That is, how fast in time can be the input polarization fluctuations of the beam, so that the Omnipolarizer still maintains its ability to function as polarizer or discrete PBS? Our equipment allowed us to drop the characteristic time of input polarization fluctuations down to 30 microseconds, without any detectable changes in the SOP stabilization performance of the Omnipolarizer. This response time is comparable with the record value of today's polarization controllers based on LithiumNiobate waveguides ${ }^{1,2}$. In Ref. 24 we have analysed the response time of another kind of typical fiber-optic nonlinear lossless polarizer based on the interaction between counter-propagating beams, and found that it is of the order of $\mathrm{L}_{\mathrm{NL}} / \mathrm{c}$, where $\mathrm{L}_{\mathrm{NL}}=(\gamma \mathrm{P})^{-1}$ is the nonlinear length, $\gamma$ is the fiber nonlinear coefficient and $\mathrm{P}$ is the input signal power. This estimate suggests that for typical operational regimes, the response time is of a few microseconds. The physical reason why the response time of the Omnipolarizer turns out to be much slower than the response time of the Kerr effect in silica (which is of the order of a few femtoseconds) is the counter-propagating nature of this nonlinear polarizer and thus the distributed interaction along the entire fiber length. Hence a finite response time is necessary in order to establish an equilibrium polarization pattern inside the nonlinear medium. In practice, this analysis suggests that the 
response time of the Omnipolarizer could be reduced by at least an order of magnitude by utilizing fibers with higher nonlinearities, such as Chalcogenide, Tellurites, Bismuth or lead Silicate fibers ${ }^{31-37}$.

The counter-propagative configuration and four-wave mixing interaction of the Omnipolarizer also impose high average powers for both forward and backward light beam, typically around $500 \mathrm{~mW}$ in Figs. 4 and 5, which is high above the standard telecommunication levels of powers and consequently, which could not be applicable to weak input signals. Nevertheless, this drawback could be also overcome by the use of highly nonlinear fiber based on soft glass materials ${ }^{31-37}$, which could allow to decrease the required average power by two orders of magnitude thanks to their strong Kerr coefficients. Another important issue for telecommunication applications is the compatibility of the Omnipolarizer with a wavelength division multiplexing (WDM) configuration. Currently, we could emphasize that because of the strong nonlinear regime of propagation occurring in the Omnipolarizer, the power level should be yet higher in a WDM configuration and thus a substantial nonlinearitymediated cross-talk would be observed between the different channels, providing a non-negligible amount of quality impairments. In order to overcome these impairments and excess of power, we would emphasize that a demultiplexing operation should be required before repolarization process in a WDM environment. This last point is presently under study and requires further investigations.

In summary, this work reports the first experimental observation of self-organization of light state of polarization in optical fiber. The device called Omnipolarizer is based on a nonlinear interaction through a four-wave-mixing process occurring in optical fibers between a signal beam and its own counter-propagating replica produced by back-reflection at the fiber output end. Two modes of operation of the Omnipolarizer were experimentally demonstrated. First as digital circular beam splitter when only a reflective element such as FBG was inserted at the end of the fiber. This configuration allowed us to successfully route an arbitrary polarized OOK 40-Gbit/ $\mathrm{s}$ signal into a well-defined universal right or left circular SOP without any polarization depending loss induced RIN. Secondly as an ideal polarizer, it corresponds to the implementation of an amplified feedback loop at the end of the fiber. In this configuration, the Omnipolarizer has allowed us to self-trap the state of polarization of an arbitrary polarized OOK 40-Gbit/s without any polarization depending loss, and provided weak penalty when compared to backto-back measurements. In conclusion, our observations of polarization self-organization in a standard telecommunication optical fiber unveil the possibility for a radically new approach to light polarization control, and open up the path to new exciting applications in photonics including signal processing, imaging, laser, spintronics or sensing measurements.

\section{Methods}

The 40-Gbit/s Return-to-Zero signal was generated by means of a 10-GHz modelocked fiber laser (Calmar Laser) delivering 2.5-ps pulses at $1564 \mathrm{~nm}$. The spectrum of the initial pulse train was frequency sliced by means of a programmable liquidcrystal based optical filter (Finisar Waveshaper) in order to enlarge the pulses to 7.5 -ps Gaussian pulses. The resulting $10-\mathrm{GHz}$ pulse train was intensity modulated by a $\mathrm{LiNbO}_{3}$ modulator through a $2^{31}-1$ pseudo-random bit sequence PRBS (Modbox Photline technologies and Anritsu pattern generator) before $\mathrm{x} 4$ time multiplexing to achieve a 40-Gbit/s optical data stream. A polarization scrambler (Agilent polarimeter) was then used to introduce wide polarization fluctuations at a rate of $0.625 \mathrm{kHz}$. Before injection into the optical fiber, the 40-Gbit/s signal was finally amplified by means of an Erbium doped fiber amplifier (EDFA from Manlight) at an average power of $27 \mathrm{dBm}$. The optical fiber within the Omnipolarizer was a $6.2-\mathrm{km}$ long standard Non-Zero Dispersion-Shifted Fiber (NZDSF) with chromatic dispersion $\mathrm{D}=-1.5 \mathrm{ps} / \mathrm{nm} / \mathrm{km}$ at $1550 \mathrm{~nm}$, a dispersion slope $\mathrm{S}=0.07 \mathrm{ps}^{2} / \mathrm{nm} / \mathrm{km}$, a nonlinear Kerr coefficient $\gamma=1.7 \mathrm{~W}^{-1} \mathrm{~km}^{-1}$ and a PMD coefficient $\mathrm{D}_{\mathrm{p}}=0.05 \mathrm{ps} /$ $\mathrm{km}^{1 / 2}$. An optical circulator was inserted at the input of the fiber. At the opposite end of the fiber, two configurations were tested. The first one involved a FBG with a 1-THz flat-top bandwidth centred at the signal wavelength with a reflection (transmission) ratio of $95 \%$ (5\%), respectively. After propagation, the repolarized $40-\mathrm{Gbit} / \mathrm{s}$ data signal was optically filtered by means of a $70-\mathrm{GHz}$ Gaussian shape optical bandpass filter (programmable WaveShaper filter from Finisar) shifted by $290 \mathrm{GHz}$ from the initial signal central frequency so as to improve the intensity extinction ratio ${ }^{20}$. At the receiver, a polarizer or an association of quarter-wave plate and linear PBS, were inserted in order to translate the polarization fluctuations into intensity fluctuations. Behind the polarizer, the $40-\mathrm{Gbit} / \mathrm{s}$ eye diagram was monitored by an optical sampling oscilloscope (OSO EXFO picosolve) while the data were detected by means of a 70-GHz photodiode (from $\mathrm{u} 2 \mathrm{t}$ ) and electrically demultiplexed (Centellax 56Gbit/s demultiplexer) at $10 \mathrm{Gbit} / \mathrm{s}$ in order to measure the bit error rate (BER). Note that the BER measurements represented in Fig. 5 are averaged on the 4 resulting demultiplexed 10-Gbit/s channels. The 40-Gbit/s signal SOP was also analyzed by means of a commercially available polarization analyzer (Agilent polarimeter).

1. Martinelli, M., Martelli, P. \& Pietralunga, S. M. Polarization stabilization in optical communications systems. J. Lightw. Technol. 24, 4172-4183 (2006).

2. Koch, B., Noe, R., Mirvoda, V., Griesser, H., Bayer, S. \& Wernz, H. Record 59-krad/s Polarization Tracking in 112-Gb/s 640-km PDM-RZ-DQPSK Transmission. IEEE Photonics Technol. Lett. 22, 1407-1409 (2010).

3. Mujat, M. \& Dogariu, A. Polarimetric and spectral changes in random electromagnetic fields. Optics Lett. 28, 2153-2155 (2003).

4. Sorrentini, J., Zerrad, M., Soriano, G. \& Amra, C. Enpolarization of light by scattering media. Optics Express 19, 21313-21320 (2011).

5. Svirko, Yu. P. \& Zheludev, N. I. Propagation of partially polarized light. Phys. Rev. A 50, 709-713 (1994)

6. Svirko, Yu. P. \& Zheludev, N. I. A new principle of broken time reversibility in solids using unpolarized light. J. of Luminiscence 58, 399-402 (1994).

7. Prakash, H. \& Singh, D. K. Change in coherence properties and degree of polarization of light propagating in a lossless isotropic nonlinear Kerr medium. J. Phys. B: At. Mol. Opt. Phys. 41, 045401 (2008).

8. Picozzi, A. Spontaneous polarization induced by natural thermalization of incoherent light. Opt. Express 16, 17171 (2008).

9. Connaughton, C., Josserand, C., Picozzi, A., Pomeau, Y. \& Rica, S. Condensation of Classical Nonlinear Waves. Phys. Rev. Lett. 95, 263901 (2005).

10. James, D. F. V. Change of polarization of light beams on propagation in free space. J. Opt. Soc. Am. A 11, 1641-1643 (1994).

11. Thevenaz, L., Zadok, A., Eyal, A. \& Tur, M. All-optical polarization control through Brillouin amplification. in Optical Fiber Communication Conference, OFC'08, paper OML7 (2008).

12. Zadok, A., Zilka, E., Eyal, A., Thévenaz, L. \& Tur, M. Vector analysis of stimulated Brillouin scattering amplification in standard single-mode fibers. Opt. Express 16, 21692-21707 (2008).

13. Galtarossa, A., Palmieri, L., Santagiustina, M., Schenato, L. \& Ursini, L. Polarized Brillouin Amplification in Randomly Birefringent and Unidirectionally Spun Fibers. IEEE Photon. Technol. Lett. 20, 1420-1422 (2008).

14. Shmilovitch, Z., Primerov, N., Zadok, A., Eyal, A., Chin, S., Thevenaz, L. \& Tur, M. Dual-pump push-pull polarization control using stimulated Brillouin scattering. Opt. Express 19, 25873-25880 (2011).

15. Martinelli, M., Cirigliano, M., Ferrario, M., Marazzi, L. \& Martelli, P. Evidence of Raman-induced polarization pulling. Opt. Express 17, 947-955 (2009).

16. Kozlov, V. V., Nuño, J., Ania-Castañón, J. D. \& Wabnitz, S. Theory of fiber optic Raman polarizers. Opt. Lett. 35, 3970-3972 (2010).

17. Ursini, L., Santagiustina, M. \& Palmieri, L. Raman Nonlinear Polarization Pulling in the Pump Depleted Regime in Randomly Birefringent Fibers. IEEE Photon. Technol. Lett. 23, 1041-1135 (2011).

18. Muga, N. J., Ferreira, M. F. S. \& Pinto, A. N. Broadband polarization pulling using Raman amplification. Opt. Express 19, 18707-18712 (2011).

19. Fatome, J., Pitois, S., Morin, P. \& Millot, G. Observation of light-by-light polarization control and stabilization in optical fibre for telecommunication applications. Opt. Express 18, 15311-15317 (2010).

20. Morin, P., Fatome, J., Finot, C., Pitois, S., Claveau, R. \& Millot, G. All-optical nonlinear processing of both polarization state and intensity profile for $40 \mathrm{Gbit} / \mathrm{s}$ regeneration applications. Opt. Express 19, 17158-17166 (2011).

21. Pitois, S., Picozzi, A., Millot, G., Jauslin, H. R. \& Haelterman, M. Polarization and modal attractors in conservative counterpropagating four-wave interaction. Europhysics Letters 70, 88 (2005).

22. Kozlov, V. V., Turitsyn, K. \& Wabnitz, S. Nonlinear repolarization in optical fibers: polarization attraction with copropagating beams. Opt. Lett. 36, 4050 (2011).

23. Heebner, J. E., Bennink, R. S., Boyd, R. W. \& Fisher, R. A. Conversion of unpolarized light to polarized light with greater than $50 \%$ efficiency by photorefractive two-beam coupling. Opt. Lett. 25, 257-259 (2000).

24. Kozlov, V. V., Fatome, J., Morin, P., Pitois, S., Millot, G. \& Wabnitz, S. Nonlinear repolarization dynamics in optical fibers: transient polarization attraction. J. Opt. Soc. Am. B 28, 1782-1791 (2011).

25. Kozlov, V. V., Javier Nuno \& Wabnitz, S. Theory of lossless polarization attraction in telecommunication fibers. J. Opt. Soc. Am. B 28, 100-108 (2011).

26. D'Alessandro, G. \& Firth, W. J. Spontaneous hexagon formation in a nonlinear optical medium with feedback mirror. Phys. Rev. Lett. 66, 2597-2600 (1991).

27. Assémat, E., Picozzi, A., Jauslin, H. \& Sugny, D. Hamiltonian tools for the analysis of optical polarization control. J. Opt. Soc. Am. B 29, 559 (2012).

28. Cushman, R. H. \& Bates, L. M. Global aspects of classical integrable systems. Birkhauser, Berlin (1997). 
29. Sugny, D., Picozzi, A., Lagrange, S. \& Jauslin, H. R. On the role of singular tori in the spatio-temporal dynamics of nonlinear wave systems. Phys. Rev. Lett. 103, 034102 (2009).

30. Assémat, E., Lagrange, S., Picozzi, A., Jauslin, H. R. \& Sugny, D. Complete nonlinear polarization control in an optical fiber system. Opt. Lett. 35, 2025-2027 (2010).

31. Granzow, N., Stark, S. P., Schmidt, M. A., Tverjanovich, A. S., Wondraczek, L. \& Russell, P. St. J. Supercontinuum generation in chalcogenide-silica step-index fibers. Opt. Express 19, 21003-21010 (2011).

32. Liao, M., Chaudhari, C., Qin, G., Yan, X., Kito, C., Suzuki, T., Ohishi, Y., Matsumoto, M. \& Misumi, T. Fabrication and characterization of a chalcogenidetellurite composite microstructure fiber with high nonlinearity. Opt. Express 17, 21608-21614 (2009).

33. Lamont, M. R., Luther-Davies, B., Choi, D.-Y., Madden, S. \& Eggleton, B. J. Supercontinuum generation in dispersion engineered highly nonlinear $(\gamma=10$ / $\mathrm{W} / \mathrm{m}) \mathrm{As}_{2} \mathrm{~S}_{3}$ chalcogenide planar waveguide. Opt. Express 16, 14938-14944 (2008).

34. Domachuk, P., Wolchover, N. A., Cronin-Golomb, M., Wang, A., George, A. K., Cordeiro, C. M. B., Knight, J. C. \& Omenetto, F. G. Over $4000 \mathrm{~nm}$ bandwidth of mid-IR supercontinuum generation in sub-centimeter segments of highly nonlinear tellurite PCFs. Opt. Express 16, 7161-7168 (2008).

35. Conseil, C., Coulombier, Q., Boussard-Pledel, C., Troles, J., Brilland, L., Renversez, G., Mechin, D., Bureau, B., Adam, J. L. \& Lucas, J. Chalcogenide step index and microstructured single mode fibers. J. Non-Cryst. Solids 357, 2480-2483 (2011).

36. Feng, X., Poletti, F., Camerlingo, A., Parmigiani, F., Petropoulos, P., Horak, P., Ponzo, G. M., Petrovich, M., Shi, J. D., Loh, W. H. \& Richardson, D. J. Dispersion controlled highly nonlinear fibers for all optical processing at telecoms wavelengths. Opt. Fib. Technol. 16, 378-391 (2010).

37. El-Amraoui, M., Gadret, G., Jules, J. C., Fatome, J., Fortier, C., Désévédavy, F., Skripatchev, I., Messaddeq, Y., Troles, J., Brilland, L., Gao, W., Suzuki, T., Ohishi, Y. \& Smektala, F. Microstructured chalcogenide optical fibers from As2S3 glass: towards new IR broadband sources. Opt. Express 18, 26655-26665 (2010).

\section{Acknowledgements}

All the experiments were performed on the PICASSO platform in ICB. The research leading to these results has received funding from the European Research Council under the European Community's Seventh Framework Programme (FP7/2007-2013 Grant Agreement $n^{\circ} 306633$, PETAL project coordinated by Julien Fatome). We also acknowledge the financial support from the CNRS, Conseil Régional de Bourgogne: Photcom PARI program, Ministère de l'Enseignement Supérieur et de la Recherche and FEDER. The work of V. K. and S. W. was carried out with support from the Italian Ministry of Research and University (MIUR) through grant 2008MPSSNX.

\section{Author contributions}

J.F. and S.P. provided equal contributions. J.F., S.P. and P.M. carried out the experiments. D.S., A.P., E.A., H.J., V.K. and S.W. contributed to the theoretical and numerical analysis. S.W. provided overall technical leadership. G.M. provided supervision. All authors participated in the analysis of the results and in the paper redaction.

\section{Additional information}

Supplementary Information accompanies this paper at http://www.nature.com/ scientificreports

Competing financial interests: The authors declare no competing financial interests License: This work is licensed under a Creative Commons Attribution-NonCommercial-NoDerivs 3.0 Unported License. To view a copy of this license, visit http://creativecommons.org/licenses/by-nc-nd/3.0/

How to cite this article: Fatome, J. et al. A universal optical all-fiber omnipolarizer. Sci. Rep. 2, 938; DOI:10.1038/srep00938 (2012) 